Published in final edited form as:

Ruprecht, C., Geißner, A., Seeberger, P. H., \& Pfrengle, F. (2019). Practical considerations for printing high-density glycan microarrays to study weak carbohydrate-protein interactions. Carbohydrate Research, 481, 31-35. doi:10.1016/j.carres.2019.06.006.

\title{
Practical considerations for printing high-density glycan microarrays to study weak carbohydrate- protein interactions
}

Colin Ruprecht, Andreas Geissner, Peter H. Seeberger, Fabian Pfrengle
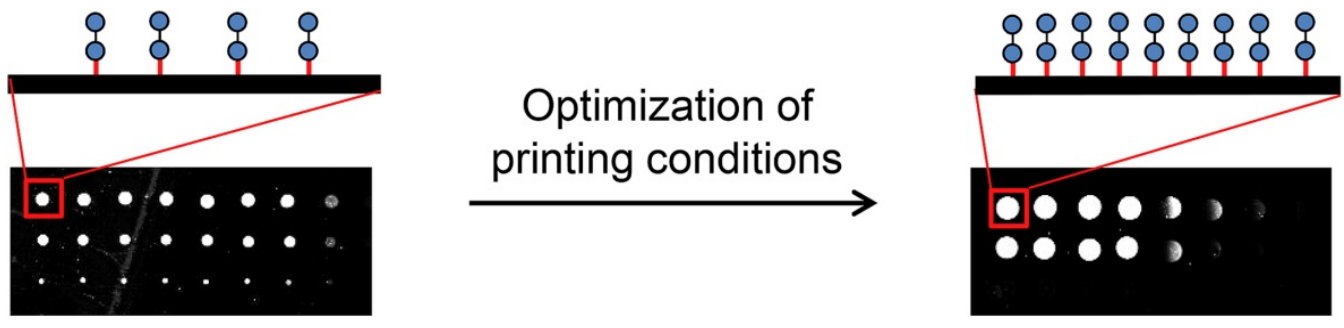

Key words:

glycan microarrays, glycan-binding proteins, immobilization of glycans, glycan-protein interactions 
Title: Practical considerations for printing high-density glycan microarrays to study weak carbohydrate-protein interactions

Colin Ruprecht ${ }^{1}$, Andreas Geissner ${ }^{1,2,3}$, Peter H. Seeberger ${ }^{1,2}$, Fabian Pfrengle ${ }^{1,2 *}$

\title{
Affiliations
}

${ }^{1}$ Department of Biomolecular Systems, Max Planck Institute of Colloids and Interfaces, Am Mühlenberg 1, 14476 Potsdam, Germany

${ }^{2}$ Institute of Chemistry and Biochemistry, Freie Universität Berlin, Arnimallee 22, 14195

Berlin, Germany

${ }^{3}$ Present address: Department of Chemistry, University of British Columbia, Vancouver, British Columbia, Canada

*Corresponding author: Fabian.Pfrengle@mpikg.mpg.de, Phone: +49 3315679357

Key words: glycan microarrays, glycan-binding proteins, immobilization of glycans, glycanprotein interactions

\begin{abstract}
Interactions of carbohydrates and proteins are essential for many biological processes and glycan microarrays have emerged as powerful tools to rapidly assess these carbohydrateprotein interactions. Diverse platforms to immobilize glycans on glass slides for subsequent probing of the specificities of glycan-binding proteins (GBPs) have evolved. It has been suggested that high local glycan density on microarrays is crucial for detecting low-affinity interactions. To determine the influence of printing efficacy on GBP binding, we compared $\mathrm{N}$-hydroxyl succinimide (NHS)-ester activated glass slides from three different manufacturers and evaluated two different printing buffers. Large differences in binding efficacies of Concanavalin A, peanut agglutinin, and Ricinus communis agglutinin 120 were observed. On some slides, low affinity interactions were missed altogether. Addition of polyethylenglycol (PEG) 400 to the printing buffer significantly enhanced the sensitivity of the binding assays. After monitoring printing efficacy over prolonged printing times, substantial effects resulting from progressing hydrolysis of the NHS-esters during the printing run on one type of slides were found. Printing efficiency of glycans strongly depends on the type of NHS-ester activated slides, the printing buffer, and the printing time. We provide practical advice for selecting the right printing conditions for particular applications.
\end{abstract}




\section{Introduction}

Glycan-binding proteins (GBPs), such as antibodies and lectins, play important roles in many aspects of life, including cell-cell interaction, cancer recognition by the human immune system, host-pathogen interaction, and innate immunity in animals and plants [1-3]. The binding specificities of GBPs can be determined in high throughput using glycan microarrays that contain carbohydrates immobilized on glass slides for subsequent interrogation of the binding events [4, 5]. Picoliter amounts of glycan solutions are printed using specialized printing robots. Since their introduction in 2002, glycan microarrays have been used to analyze the specificities of bacterial, animal, plant and human GBPs, and antibodies [6-16]. Several different glycan array platforms have been developed. Mostly, direct immobilization using a functionalized linker on the carbohydrate and indirect immobilization via printing of glycan-protein conjugates is employed [5, 17, 18]. A previous cross-comparison of glycan array platforms revealed differences regarding the detection sensitivities of carbohydrateGBP interactions, probably resulting from differences in local glycan densities. These differences significantly impact the measurement of weak binding interactions [19]. The glycan array from the Consortium of Functional Glycomics, containing more than 600 mammalian oligosaccharides, is most widely used by the scientific community and is printed through direct immobilization of the glycans on $N$-hydroxyl succinimide (NHS) esteractivated glass slides [17]. GBP-binding to several ligands was missed on this array when compared to the Gildersleeve arrays that immobilize protein-linked carbohydrates and therefore may result in higher local glycan densities [19]. NHS-ester chemistry is the method of choice for many labs due to the ease of glycan immobilization. Existing microarray platforms that use NHS-ester chemistry for glycan immobilization print the oligosaccharides on the activated glass slides differently. Different labs successfully use slides from different manufacturers, for example Nexterion H slides from Schott [8, 20, 21], Codelink slides from Surmodics [8, 22], and less commonly also slides from the manufacturer PolyAn [23, 24]. Moreover, different printing buffers such as sodium phosphate buffers or phosphate buffers including polyethylenglycol (PEG) 400 [12, 17, 25, 26], different humidity [18, 27], and different glycan concentrations are used for printing [19]. In order to improve printing efficiency and optimize glycan density on microarrays using direct immobilization via NHSester chemistry, we analyzed the influence of printing conditions, including the printing buffer and humidity, while printing on different commercially available microarray slides on GBP-binding. 
A
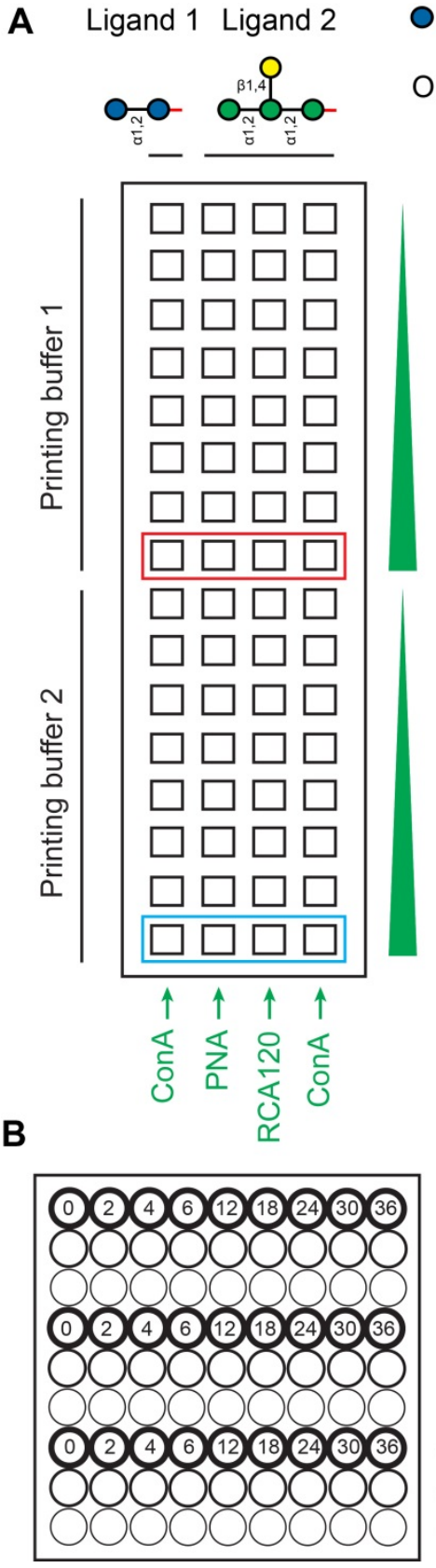

Glucose O Mannose $\bigcirc$ Galactose

Oligosaccharide linker $-=$

C

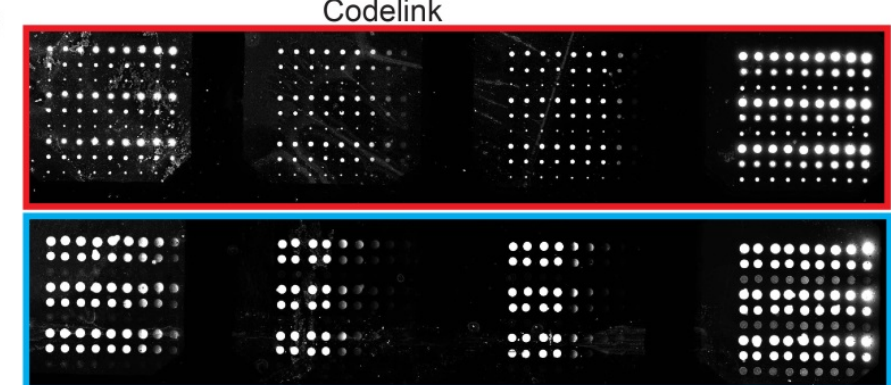

PB1

PB2

Nexterion $\mathrm{H}$

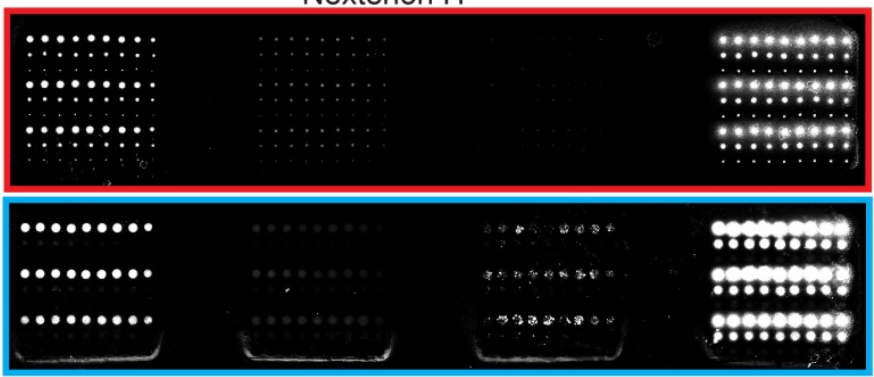

PB1

PB2

PolyAn

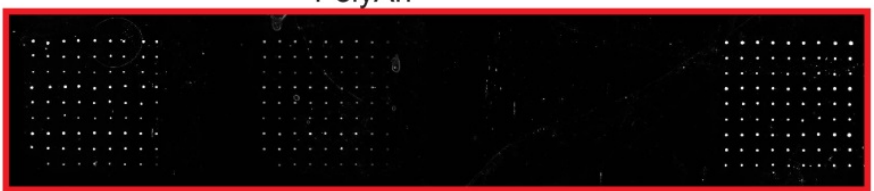

PB1

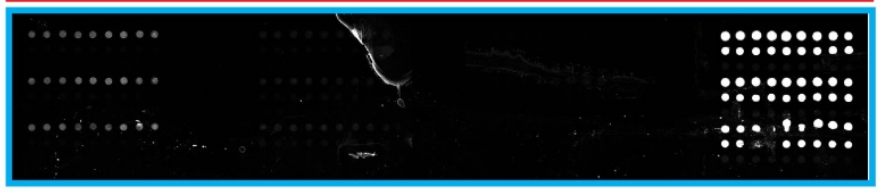

PB2
ConA
PNA
RCA120
ConA
ligand1
ligand2

Figure 1. Experimental setup to assess printing efficacy.

(A) Design of microarray slide with 64 fields. Ligand 1 was printed in the fields of the first column, and ligand 2 was printed in the remaining three columns. The upper half of fields was printed with printing buffer (PB) 1:

50mM NaH2PO4 pH 8.5; the lower half was printed with PB2: 80\% 50mM NaH2PO4 pH 8.5, 0.005\% CHAPS, $20 \%$ PEG400. After printing, the slides were probed with peanut agglutinin (PNA), Ricinus communis agglutinin 120 (RCA120) and ConcanavalinA (ConA), as indicated in green below the slide. Note that ConA was probed against ligand 1 and ligand 2. For each GBP, a 1:3 dilution series with the highest concentration of $0.2 \mathrm{mg} / \mathrm{ml}$ was used. (B) Printing pattern for each field with three different printing concentrations (10-400 $\mu \mathrm{M})$ and nine time points from 0-36 h, with the first printed spots at $0 \mathrm{~h}$ and the last printed spots at 36h. Each spot was printed in triplicates. (C) Scans of fields that are indicated in red and blue in (A) on the three types of slides printed with PB1 (red) or PB2 (blue) at 40\% humidity, and probed with ConA, PNA, and RCA120 (0.2 mg/ml) as GBP. All images were obtained using the same photomultiplier gain and exported from the GenePix program using the same settings for brightness and contrast. 


\section{Results and Discussion}

In a previous study we have employed a synthetic plant glycan microarray for characterizing the epitopes of plant cell wall glycan-directed monoclonal antibodies [12]. We noticed that certain expected binding events were missed on Codelink slides when using standard printing conditions (Figure S1). After changing several parameters, such as humidity and printing buffer, we had found printing conditions that led to stronger signals for antibody binding without affecting the background and thus met our needs. To analyze the influence of printing conditions on GBP-binding more systematically, we devised an experimental setup to simultaneously test two different printing buffers and three printing concentrations over a time-course of $36 \mathrm{~h}$ on different types of slides (Figure 1). We obtained $N$-hydroxyl succinimide (NHS)-activated glass slides from three manufacturers: CodeLink slides from Surmodics (Eden Prairie, MN, USA), Nexterion H slides from Schott (Mainz, Germany), and 3D-NHS slides from PolyAn (Berlin, Germany). Two oligosaccharides were printed at three different humidities (40,50, and 60\%) to monitor putative hydrolysis of the NHS-esters during longer printing runs. To compare high- and low-affinity GBP-carbohydrate interactions, we printed two different oligosaccharides as ligands: an $\alpha-1,2$ linked diglucoside (ligand 1 [28]) and an $\alpha-1,2$ linked trimannoside substituted at the central mannose with a $\beta$ 1,4 linked galactosyl residue (ligand 2, Figure 1A [29]). Each spot was printed in triplicates to assess the technical variance of the printing conditions. The immobilized glycans were subsequently probed with the fluorescein-labeled GBPs Concanavalin A (ConA, mediumaffinity GBP for ligand 1 and high-affinity GBP for ligand 2), peanut agglutinin (PNA), and Ricinus communis agglutinin (RCA) 120 (both low affinity GBPs for ligand 2, [30, 31], using a series of eight $1: 3$ dilutions, starting with $0.2 \mathrm{mg} / \mathrm{mL}$ as the highest concentration (Figure 1).

With this experimental setup, we found distinct strengths of GBP binding signals depending on the printing conditions (Figure 1C). Using the fluorescence signal of the bound GBPs as a rough approximation for glycan density, we observed higher printing efficacy on CodeLink slides compared to Nexterion $\mathrm{H}$ and PolyAn slides (Figure 2A). Moreover, we observed that including 20\% PEG400 in the printing buffer (PB2) increased GBP binding compared to plain sodium phosphate buffer (PB1) on all slide types, with CodeLink slides showing the largest differences (Figure 2A). PEG, a strongly hydrophilic compound, enhances the hydrophilicity of membranes and surfaces [32-34]. We hypothesize that PEG400 increases the hydrophilicity of the polymeric surface layer coating the glass slides, resulting in improved accessibility of the reactive NHS-esters to the oligosaccharides and ultimately higher glycan densities. For example, the low-affinity interaction between GBP RCA120 and ligand 2 could be detected on Codelink slides with both printing buffers, while on Nexterion $\mathrm{H}$ slides RCA120 binding was only observed when the PEG400 containing PB2 was used (Figure 2A and B, Figure S2). 
A

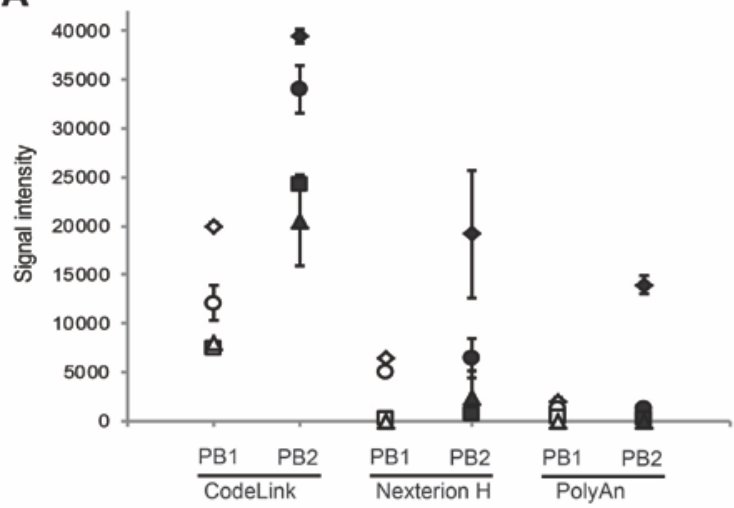

$\begin{array}{llll}\bigcirc & \text { ConA Ligand 1 } & \square & \text { PNA } \\ \triangle & \text { RCA120 } & \diamond & \text { ConA Ligand 2 }\end{array}$
B

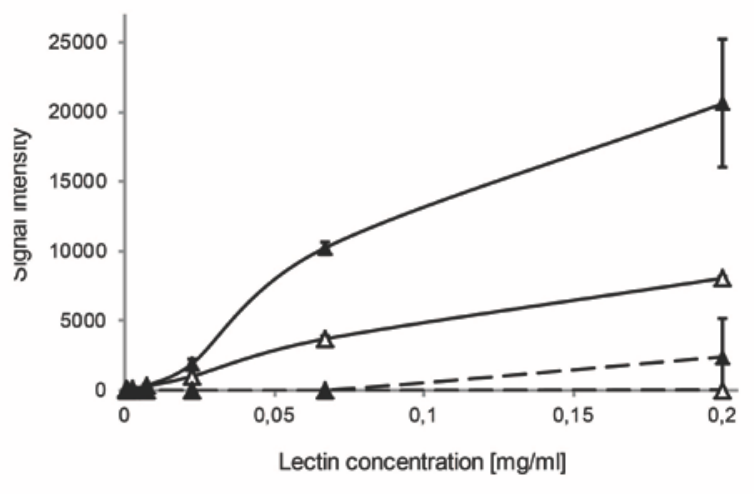

$\begin{array}{ll}\text { Printing Buffer } 1 & - \text { CodeLink } \\ \text { Printing Buffer 2 } & -\end{array}$

Fig. 2. Printing efficacy on slides from different manufacturers after using different printing buffers.

(A) Binding of ConA, PNA, and RCA120 to the respective oligosaccharides on different types of microarray slides. For each slide type the influence of the two printing buffers on subsequent GBP binding is displayed. (B) RCA120 binding after printing on CodeLink (solid line) and Nexterion H slides (dashed line). Note that for the Nexterion H slides RCA120 binding was only observed when PB2 was used for printing (filled symbol). Printing was performed at $40 \%$ humidity. Unless indicated differently, the highest ligand concentration (400 $\mu \mathrm{M})$, highest GBP concentration $(0.2 \mathrm{mg} / \mathrm{ml})$, and the first time point $(0 \mathrm{~h})$ are displayed for the data points. Error bars indicate the standard deviation of printed technical triplicates.

A
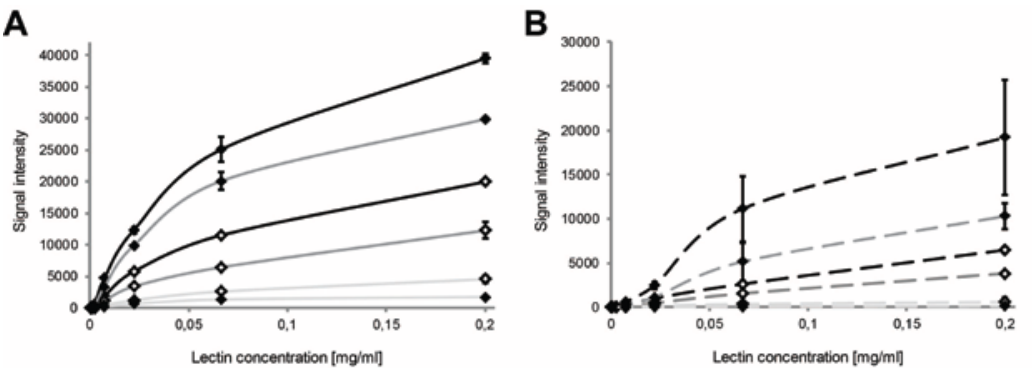

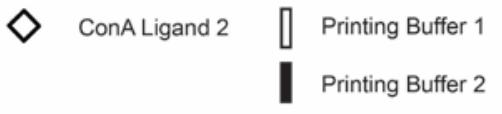

Lectin concentration $[\mathrm{mg} / \mathrm{ml}]$

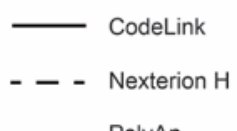

PolyAn

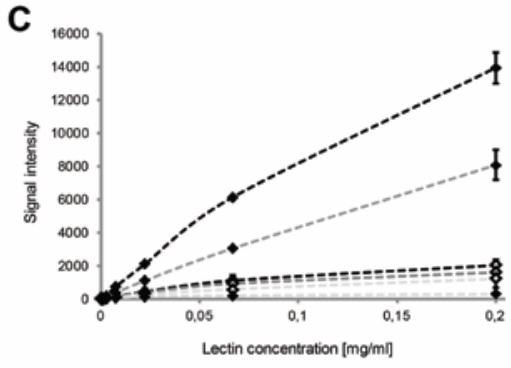

$400 \mu \mathrm{M}$ printing concentration

$100 \mu \mathrm{M}$ printing concentration

$10 \mu \mathrm{M}$ printing concentration

Fig. 3. Influence of the printing concentration and buffer on printing efficacy.

Binding of ConA to ligand 2 printed in three different concentrations (10 $\mu \mathrm{M}$ light grey, $100 \mu \mathrm{M}$ grey, and 400 $\mu \mathrm{M}$ black lines). Note that data points for the glycan printed with PB1 (open symbols) are in between the $10 \mu \mathrm{M}$ and $100 \mu \mathrm{M}$ concentrations printed with PB2 (filled symbols) for Codelink slides (A), Nexterion H slides (B) and PolyAn slides (C). Data for slides printed at $40 \%$ humidity is shown. 
Next, we analyzed the influence of glycan concentration $(10 \mu \mathrm{M}, 100 \mu \mathrm{M}$ and $400 \mu \mathrm{M})$ during printing on glycan density on the microarray. We found that increasing the glycan concentration yielded higher glycan density on the array on all slide types and for both printing buffers (Figure 3). However, the extend to which glycan concentration correlated with glycan density was dependent on the slide type and the printing buffer. Relatively similar glycan densities were obtained when printing different glycan concentrations using PB1, whereas the glycan density correlated more with the printed oligosaccharide concentration for PB2 (Figure 3). Thus, the addition of PEG400 to the printing buffer does only result in higher local glycan densities when high printing concentrations are used. We noticed that the printed drop was evaporating relatively quickly on the slide during printing when PB1 was used, while the PEG400 in PB2 strongly reduced the rate of evaporation. During evaporation, the oligosaccharide concentration increases, thus resulting in more efficient reactions of the oligosaccharides with the NHS esters on the slide, especially at low printing concentrations. This hypothesis was further supported by the smaller sizes of spots for lower glycan concentrations when printing with PB1, while more similar spot sizes were obtained for the three glycan concentrations when printing with PB2 (Figure 1C). When we analyzed plant glycan-directed antibodies we found a similar effect of the two printing buffers on spot size and glycan density when using four different printing concentrations (Figure S1F).

To test hydrolysis rates of the NHS esters during the printing runs, we monitored glycan printing efficacies over a time period of $36 \mathrm{~h}$. Printing efficacies on CodeLink slides decreased with prolonged printing times, as detected by decreased ConA binding to ligand 1 and RCA120 binding to ligand 2 (Figure 4A and C). Increasing humidity during printing (from 40 to 60\%) strongly enhanced this effect for ConA binding to ligand 1 (Figure 4B, Figure S3), indicating that humidity is the major cause for the reduced printing efficacies on the CodeLink slides. A direct comparison of the slide types showed that Nexterion $\mathrm{H}$ and PolyAn slides ensure stable printing efficacies with prolonged printing times, at both $40 \%$ and 60\% humidity (Figure 4A and B). Notably, decreased printing efficacy due to NHS ester hydrolysis particularly affected low affinity binding events. The high-affinity binding of ConA to ligand 2 (Figure S2C, F, I) was less severely impacted by reduced glycan densities than the medium-affinity binding of ConA to ligand 1 (Figure 4A, B). The low-affinity binding of PNA and RCA120 to ligand 2 strongly decreased after 40\% humidity-printing (Figure S2A, B) and exponentially decreased over time at higher humidity (Figure S2D, E, G, $\mathrm{H})$. We had noticed similar effects for the binding of two monoclonal antibodies to $\beta-1,4-$ linked galactan oligosaccharides (Figure S1C, D). Overall, these data show that the signal intensity corresponding to bound GBP, although no linear correlation can be expected, can serve as a rough approximation for glycan density on the microarray for the investigated ligand-protein interactions. 
A

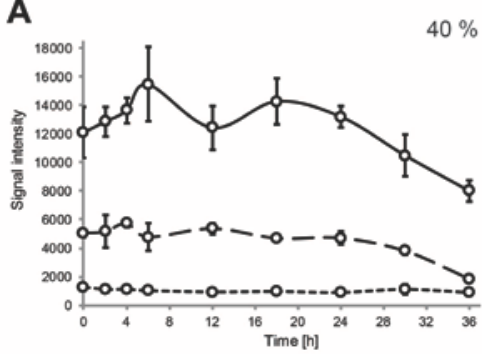

ConA Ligand 1

$\triangle$ RCA120
B

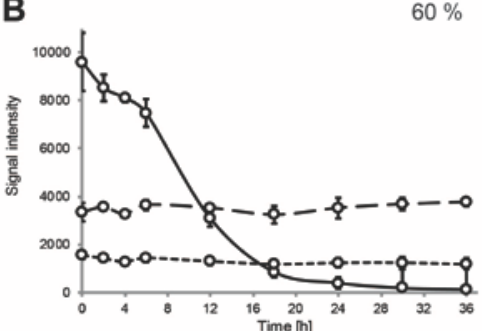

Printing Buffer 1
Printing Buffer 2
$60 \%$

C

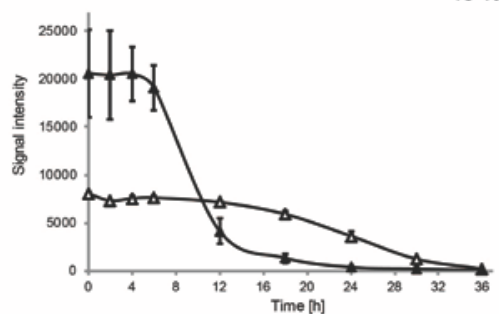

Fig. 4. Influence of humidity on printing efficacy during prolonged printing runs.

(A, B) ConA binding to ligand 1 after prolonged printing times on CodeLink (solid line), Nexterion $\mathrm{H}$ (dashed line) and PolyAn slides (dotted line). (C) RCA120 binding after printing for prolonged times on CodeLink slides. Displayed are differences between PB1 (open symbols) and PB2 (filled symbols). Printing was performed at $40 \%$ humidity (A, C) or $60 \%$ humidity (B). The highest ligand concentration (400 $\mu \mathrm{M}$ ), and the highest GBP concentration $(0.2 \mathrm{mg} / \mathrm{ml})$ are displayed for the data points.

Finally, we analyzed NHS ester hydrolysis during prolonged printing times in different printing buffers. The negative effect of NHS ester hydrolysis on printing efficacy was stronger in PB2 than in PB1 (Figure 4C, Figure S3). RCA120 binding to ligand 1 dropped steeply after $6 \mathrm{~h}$ of printing on CodeLink slides for PB2, and only gradually for PB1 (Figure 4C). This decrease in printing efficacy over time for PB2 was observed also for ConA binding to ligand 1 on CodeLink slides, albeit to a lesser extend (Figure S3A). Conversely, Nexterion $\mathrm{H}$ and PolyAn slides did not show this strong decrease in printing efficacy when printing was performed over prolonged times at $40 \%$ humidity. Only when printing was performed at 60\% humidity, a similar effect was observed for Nexterion $\mathrm{H}$ slides after $12 \mathrm{~h}$ when PB2 was used (Figure S3D). To explain this unexpected result, we hypothesize that evaporation of PB1 and the resulting increased oligosaccharide concentration compensates for an increased number of hydrolyzed NHS esters. We noticed that storage of oligosaccharides in the PEG400 containing PB2 at $4{ }^{\circ} \mathrm{C}$ or at $-20{ }^{\circ} \mathrm{C}$ led to inefficient immobilization of the oligosaccharides and permanent precipitation of buffer components. Nonetheless, when high glycan densities are required and printing times are kept short, addition of PEG400 to the printing buffer will improve printing efficacy and allow for glycan-protein interactions to be detected that are otherwise invisible. 


\section{Conclusion}

An analysis of glycan microarray printing conditions provided insights into the influence of slide type, printing buffer, oligosaccharide concentration, and length of printing run on the immobilization of oligosaccharides on NHS-activated slides. Low-affinity GBP-carbohydrate interactions may be missed on Nexterion $\mathrm{H}$ and PolyAn slides while CodeLink slides potentially provide higher signal intensities for GBP binding. Monitoring the printing efficacy over longer time periods (0-36 h) revealed that prolonged printing runs on Nexterion $\mathrm{H}$ and PolyAn slides resulted in stable signals after glycan detection with different GBPs, whereas on Codelink slides decreased GBP binding, due to hydrolysis of the NHS esters during printing, was observed. Printing runs on CodeLink slides should thus be kept short ( $<12 \mathrm{~h}$ ) and performed at low humidity, e.g. $40 \%$. The printing efficacy may be enhanced by the addition of PEG400 to the printing buffer. These results suggest that, depending on the requirements, NHS-activated slides and the printing process must be carefully selected to ensure optimal results when probing carbohydrate-GBP interactions on microarrays.

\section{Materials and Methods}

\subsection{Glycan array printing}

For our analysis we used an $\alpha-1,2$ linked diglucoside (ligand 1) and an $\alpha-1,2$ linked trimannoside substituted at the central mannose with a $\beta-1,4$ linked galactosyl residue (ligand 2, Figure 1) that have previously been chemically synthesized as described in the respective reports [28, 29]. The oligosaccharides were printed on $N$-hydroxysuccinimide (NHS) esteractivated glass slides from three different manufacturers, i.e. CodeLink slides from SurModics (Eden Prairie, MN, USA), Nexterion H slides from Schott (Mainz, Germany), and 3D-NHS slides from PolyAn (Berlin, Germany), using a non-contact piezoelectric spotting device (S3; Scienion, Berlin, Germany). The three slides were taken from freshly opened slide boxes and all printed at room temperature within one week starting with a printing run at $40 \%$ humidity, then at 50\%, and then at $60 \%$ humidity. Two different oligosaccharides were diluted to three printing concentrations $(400 \mu \mathrm{M}, 100 \mu \mathrm{M}$, and $10 \mu \mathrm{M})$ either in printing buffer 1 (PB1: $50 \mathrm{mM}$ sodium phosphate, $\mathrm{pH}$ 8.5) or in freshly prepared printing buffer 2 (PB2: 80\% (v/v) $50 \mathrm{mM}$ sodium phosphate, $\mathrm{pH}$ 8.5, 0.005\% (v/v) CHAPS, 20\% (w/v) PEG400 (Roth, Karlsruhe, Germany)). The first spots (triplicates of each of the three concentrations) were printed at time point $0 \mathrm{~h}$. Next, we printed spots in $2 \mathrm{~h}$ intervals ( $2 \mathrm{~h}, 4$ h, 6 h), then in 6 h intervals ( 12 h, 18 h, 24 h, 30 h, 36 h). During the $36 \mathrm{~h}$ printing runs the slides were left in the printing chamber at the given humidity. The oligosaccharide solutions were stored in a 384-well plate in the printing chamber and cooled to the dew point to avoid water evaporation from or condensation to the solutions in the wells while printing. Due to technical reasons the printing solutions for each time point were taken from different wells. The printing pattern for the 64 fields on the slide is depicted in Figure 1B. After printing, the microarray slides were removed from the printing chamber and incubated for $1 \mathrm{~h}$ in a box with wet tissue paper to ensure that all reactions proceeded to completion. Then the slides were quenched for $1 \mathrm{~h}$ at room temperature in $100 \mathrm{mM}$ ethanolamine, $50 \mathrm{mM}$ sodium 
phosphate, $\mathrm{pH} \mathrm{9,} \mathrm{and} \mathrm{subsequently} \mathrm{washed} \mathrm{three} \mathrm{times} \mathrm{with} \mathrm{deionized} \mathrm{water,} \mathrm{and} \mathrm{dried} \mathrm{by}$ centrifugation (300 g/min, $2 \mathrm{~min}$ ).

\subsection{Probing of glycan-binding proteins}

We used fluorescein-labeled Concanavalin A (ConA), peanut agglutinin (PNA), and Ricinus communis agglutinin (RCA) 120 (Vector Laboratories, Burlingame, CA, USA) to assess the printing efficacies. To test the three different GBPs in eight concentrations, we applied a FlexWell 64 grid (Grace Bio-Labs, Bend, OR, USA) to the slide. The slides were blocked with $1 \%(\mathrm{w} / \mathrm{v})$ bovine serum albumin (BSA) in phosphate-buffered saline (PBS) for $1 \mathrm{~h}$ at room temperature. A series of eight $1: 3$ dilutions of the GBPs $(0.2 \mathrm{mg} / \mathrm{ml}-0.091 \mu \mathrm{g} / \mathrm{ml})$ were prepared in $20 \mathrm{mM}$ HEPES, $\mathrm{pH} 7.0$, including $2 \mathrm{mM} \mathrm{CaCl}_{2}, 2 \mathrm{mM} \mathrm{MgCl}, 0.1 \mathrm{mM}$ $\mathrm{MnCl}_{2}$. To each well on the slide, $20 \mu \mathrm{GBP}$-solution was applied and incubated for $16 \mathrm{~h}$ at 4 ${ }^{\circ} \mathrm{C}$. Unbound GBP was removed by washing the slides twice for 5 min with $10 \mathrm{mM}$ HEPES, $\mathrm{pH} 7.0$ including $1 \mathrm{mM} \mathrm{CaCl}_{2}$. After removing the grid, the slides were rinsed shortly with water and dried by centrifugation at $300 \mathrm{~g} / \mathrm{min}$ for $2 \mathrm{~min}$. The fluorescence on each slide obtained after excitation with the $488 \mathrm{~nm}$ laser was scanned with a GenePix 4300A microarray scanner using the photomultiplier gain 400 (Molecular Devices, Sunnyvale, CA, USA) and quantified using the GenePix Pro 7 software (Molecular Devices).

\section{Funding}

This work was financially supported by the Max Planck Society and the German Research Foundation (DFG, Emmy Noether program PF850/1-1 to F.P.).

\section{Acknowledgements}

We thank Jonnel Jaurigue and Katrin Sellrie for technical support.

\section{Abbreviations}

ConA, Concanavalin A; PNA, peanut agglutinin; RCA120, Ricinus communis agglutinin 120; PEG, polyethylenglycol; NHS, N-hydroxyl succinimide; HEPES, 4-(2-hydroxyethyl)-1piperazineethanesulfonic acid; PB, printing buffer; GBPs, glycan-binding proteins; 


\section{References}

1. Bertozzi, C.R. and L.L. Kiessling, Chemical glycobiology. Science, 2001. 291(5512): p. 2357-64.

2. Boller, T. and S.Y. He, Innate immunity in plants: an arms race between pattern recognition receptors in plants and effectors in microbial pathogens. Science, 2009. 324(5928): p. 742-4.

3. Sharon, N. and H. Lis, History of lectins: from hemagglutinins to biological recognition molecules. Glycobiology, 2004. 14(11): p. 53R-62R.

4. Geissner, A. and P.H. Seeberger, Glycan Arrays: From Basic Biochemical Research to Bioanalytical and Biomedical Applications. Annu Rev Anal Chem (Palo Alto Calif), 2016. 9(1): p. 223-47.

5. Rillahan, C.D. and J.C. Paulson, Glycan microarrays for decoding the glycome. Annu Rev Biochem, 2011. 80: p. 797-823.

6. Fukui, S., et al., Oligosaccharide microarrays for high-throughput detection and specificity assignments of carbohydrate-protein interactions. Nat Biotechnol, 2002. 20(10): p. 1011-7.

7. Purohit, S., et al., Multiplex glycan bead array for high throughput and high content analyses of glycan binding proteins. Nat Commun, 2018. 9(1): p. 258.

8. Stowell, S.R., et al., Microbial glycan microarrays define key features of host-microbial interactions. Nat Chem Biol, 2014. 10(6): p. 470-6.

9. Wang, D., et al., Carbohydrate microarrays for the recognition of cross-reactive molecular markers of microbes and host cells. Nat Biotechnol, 2002. 20(3): p. 275-81.

10. Geissner, A., C. Anish, and P.H. Seeberger, Glycan arrays as tools for infectious disease research. Curr Opin Chem Biol, 2014. 18: p. 38-45.

11. Fangel, J.U., et al., Carbohydrate microarrays in plant science. Methods Mol Biol, 2012. 918 : p. 351-62.

12. Ruprecht, C., et al., A Synthetic Glycan Microarray Enables Epitope Mapping of Plant Cell Wall Glycan-Directed Antibodies. Plant Physiol, 2017. 175(3): p. 1094-1104.

13. Briard, J.G., et al., Cell-based glycan arrays for probing glycan-glycan binding protein interactions. Nature Communications, 2018. 9.

14. Yang, Y.Y.M., et al., Glycan Microarray-Assisted Identification of IgG Subclass Targets in Schistosomiasis. Frontiers in Immunology, 2018. 9.

15. Park, S.J. and I.J. Shin, Fabrication of carbohydrate chips for studying protein-carbohydrate interactions. Angewandte Chemie-International Edition, 2002. 41(17): p. 3180-+.

16. Fukui, S., et al., Oligosaccharide microarrays for high-throughput detection and specificity assignments of carbohydrate-protein interactions. Nature Biotechnology, 2002. 20(10): p. 1011-1017.

17. Blixt, O., et al., Printed covalent glycan array for ligand profiling of diverse glycan binding proteins. Proc Natl Acad Sci U S A, 2004. 101(49): p. 17033-8.

18. Zhang, Y., et al., An array-based method to identify multivalent inhibitors. J Am Chem Soc, 2010. 132(28): p. 9653-62.

19. Wang, L., et al., Cross-platform comparison of glycan microarray formats. Glycobiology, 2014. 24(6): p. 507-17.

20. Pedersen, H.L., et al., Versatile high resolution oligosaccharide microarrays for plant glycobiology and cell wall research. J Biol Chem, 2012. 287(47): p. 39429-38. 
21. Vuskovic, M.I., et al., Processing and analysis of serum antibody binding signals from Printed Glycan Arrays for diagnostic and prognostic applications. Int J Bioinform Res Appl, 2011. 7(4): p. 402-26.

22. Pereira, C.L., et al., Chemical Synthesis Elucidates the Immunological Importance of a Pyruvate Modification in the Capsular Polysaccharide of Streptococcus pneumoniae Serotype 4. Angew Chem Int Ed Engl, 2015. 54(34): p. 10016-9.

23. Shanthamurthy, C.D., et al., ABO Antigens Active Tri- and Disaccharides Microarray to Evaluate C-type Lectin Receptor Binding Preferences. Sci Rep, 2018. 8(1): p. 6603.

24. Gotze, S., et al., Investigation of the protective properties of glycosylphosphatidylinositolbased vaccine candidates in a Toxoplasma gondii mouse challenge model. Glycobiology, 2015. 25(9): p. 984-91.

25. Andersen, M.C., et al., Synthesis of beta-1,4-Linked Galactan Side-Chains of Rhamnogalacturonan I. Chemistry, 2016. 22(33): p. 11543-8.

26. Dallabernardina, P., et al., Automated glycan assembly of galactosylated xyloglucan oligosaccharides and their recognition by plant cell wall glycan-directed antibodies. Org Biomol Chem, 2017. 15(47): p. 9996-10000.

27. Park, S., M.R. Lee, and I. Shin, Fabrication of carbohydrate chips and their use to probe protein-carbohydrate interactions. Nat Protoc, 2007. 2(11): p. 2747-58.

28. Martin, C.E., et al., Immunological Evaluation of a Synthetic Clostridium difficile Oligosaccharide Conjugate Vaccine Candidate and Identification of a Minimal Epitope. Journal of the American Chemical Society, 2013. 135(26): p. 9713-9722.

29. Anish, C., et al., Immunogenicity and Diagnostic Potential of Synthetic Antigenic Cell Surface Glycans of Leishmania. Acs Chemical Biology, 2013. 8(11): p. 2412-2422.

30. London, J., S. Berrih, and J.F. Bach, Peanut agglutinin. I. A new tool for studying T lymphocyte subpopulations. J Immunol, 1978. 121(2): p. 438-43.

31. Podder, S.K., A. Surolia, and B.K. Bachhawat, On the specificity of carbohydrate-lectin recognition. The interaction of a lectin from Ricinus communis beans with simple saccharides and concanavalin A. Eur J Biochem, 1974. 44(1): p. 151-60.

32. Kolahchi, A.R., A. Ajji, and P.J. Carreau, Enhancing Hydrophilicity of Polyethylene Terephthalate Surface Through Melt Blending. Polymer Engineering and Science, 2015. 55(2): p. 349-358.

33. Aryanti, P.T.P., et al., Performance and characterization of PEG400 modified PVC ultrafiltration membrane. Membrane Water Treatment, 2015. 6(5): p. 379-392.

34. Long, H.P., C.C. Lai, and C.K. Chung, Polyethylene glycol coating for hydrophilicity enhancement of polydimethylsiloxane self-driven microfluidic chip. Surface \& Coatings Technology, 2017. 320: p. 315-319. 